\title{
Maintenance Scheduling and Production Control of Multiple-Machine Manufacturing Systems
}

\author{
A. Gharbi ${ }^{a}$ and J.-P. Kennéb \\ ${ }^{a}$ Automated Production Engineering Department, University of Quebec, École de technologie \\ supérieure, Production Systems Design and Control Laboratory \\ 1100, Notre Dame Street West, Montreal (Quebec), Canada, H3C 1 K3. \\ ${ }^{b}$ Mechanical Engineering Department, University of Quebec, École de technologie supérieure, \\ 1100, Notre Dame Street West, Montreal (Quebec), Canada, H3C $1 \mathrm{~K} 3$.
}

\begin{abstract}
This paper deals with the production and preventive maintenance control problem for a multiple-machine manufacturing system. The objective of such a problem is to find the production and preventive maintenance rates for the machines so as to minimize the total cost of inventory/backlog, repair and preventive maintenance. A two-level hierarchical control model is presented, and the structure of the control policy for both identical and non-identical manufacturing systems is described using parameters, referred to here as input factors. By combining analytical formalism with simulation-based statistical tools such as experimental design and response surface methodology, an approximation of the optimal control policies and values of input factors are determined. The results obtained extend those available in existing literature to cover non-identical machine manufacturing systems. A numerical example and a sensitivity analysis are presented in order to illustrate the robustness of the proposed approach. The extension of the proposed production and preventive maintenance policies to cover large systems (multiple machines, multiple products) is discussed.
\end{abstract}

Key words: Preventive Maintenance, Hierarchical Control, Flexible Manufacturing Systems, Simulation, Experimental Design, Response Surface Methodology (RSM).

\section{Introduction}

The problem of controlling manufacturing systems with unreliable machines was formulated as a stochastic control problem by Older and Suri (1980). Failure and repair processes were supposed to be described using homogeneous Markov processes. The related optimal control model falls under the category of problems studied previously by Rishel (1975). Similar investigations have resulted in the analytical solution of the one-machine one-product manufacturing system control problem obtained by Akella and Kumar (1986). In the case of nonhomogeneous Markov processes involving states and control-dependent transition rates, the control problem becomes more complex. In this sphere, Boukas and Haurie (1990) considered the 
fact that the failure probabilities of a machine depend on its age, and they added the possibility of performing preventive maintenance to the existing models. The related age-dependent set of dynamic programming equations were solved numerically for a given manufacturing system. However, with the numerical scheme presented by Boukas and Haurie (1990), it remains difficult to obtain a general structure for the optimal control of a large class of manufacturing systems. A potential way of coping with such a difficulty is to develop heuristical methods based on the reduction of the size of the considered control problem. Hence, different approaches have been proposed in the existing literature with a view to deriving simple near-optimal control policies for manufacturing systems.

The concept of hedging point policy, introduced by Kimemia and Gershwin (1983), is one of the simple ways available for finding suboptimal control policies in the production planning and maintenance scheduling of manufacturing systems. For further details on this concept, we refer the reader to the age-dependent hedging point concept presented by Boukas et al. (1995) and by Kenne and Gharbi (1999). Because of the computation of threshold levels, the derivation of suboptimal policies based on this concept seems to be difficult for a large class of manufacturing systems. Another approach is to develop hierarchical control methods based on the particular structure of the system. This can be done by using the singular perturbation approach. Such an approach mainly involves reducing the size of the control problem according to the discrepancy between the time scales of events involved. By replacing fast processes with their respective mean values, one can construct a deterministic limiting problem, which is computationally more tractable. Details on this approach can be found in Kokotovic et al. (1986), Lehoczky et al. (1991), Sethi and Zhang (1994) and Soner (1993). In this paper, we will first define the structure of the optimal control policies, both for identical and for non-identical machine manufacturing systems. Based on such structures, we will then extend the production and maintenance rates control model presented in Kenne and Boukas (2003) in order to determine the control policy in a more general case including non-identical machine manufacturing systems. The resulting structure is described through a set of parameters we call input factors. We resort to a combination of analytical and simulation-based experimental approaches to find an approximation of the optimal control policies for production and preventive maintenance by determining the values of input factors.

In the proposed approach, the parameterized near-optimal control policy is used as an input for the simulation model. For each entry consisting of a combination of parameters, the cost incurred is obtained. It is from this relationship that the best control factor values are determined and a relationship between input factors and such a cost is given. The application of such an approach is motivated by the works of Kenne and Gharbi (1999) and Gharbi and Kenne (2000). We refer the reader to these works for a literature review on the applications of simulation and statistical methods such as experimental design and RSM in the sphere of manufacturing systems control.

The remainder of the paper is organized as follows: In section 2, the optimal control problem is described both for identical and non-identical machine systems. The proposed control approach is described in section 3. The logic of the simulation model is described in section 4. In section 5, the experimental design approach and response surface methodology are outlined. A numerical example and a sensitivity analysis are also presented in section 5. Concluding remarks are presented in section 6 . 


\section{Problem Statement}

In this section, we present an explicit formulation of the stochastic optimal control problem related to the production control and preventive maintenance scheduling of manufacturing systems with non-identical machines. Based on the large size of the optimality conditions obtained, we next present a singular perturbation form of the control model for multiple identical machine manufacturing systems. The structure of the control policy in such a situation is extended to define that policy in the case of non-identical machines, for which optimality conditions are difficult to solve.

The system under study consists of $m$ machines producing $n$ different part types. The operational mode of machine $i$ can be described by a stochastic process $\zeta_{\mathrm{i}}(\mathrm{t})(1 \leq \mathrm{i} \leq \mathrm{m})$. Such a machine is available when it is operational $\left(\zeta_{\mathrm{i}}(\mathrm{t})=1\right)$ and unavailable when it is under repair $\left(\zeta_{\mathrm{i}}(\mathrm{t})=2\right)$ or under preventive maintenance $\left(\zeta_{\mathrm{i}}(\mathrm{t})=3\right)$. We then have $\zeta_{\mathrm{i}}(\mathrm{t}) \in \mathrm{B}_{\mathrm{i}}=\{1,2,3\}$. We can describe the manufacturing system mode by the random vector $\zeta(t)=\left(\zeta_{1}(t), \cdots, \zeta_{m}(t)\right)^{\prime}$ with values in $\mathrm{B}=\mathrm{B}_{1} \times \cdots \times \mathrm{B}_{\mathrm{m}}$.

Let $\mathrm{a}=\left(\mathrm{a}_{1}, \cdots, \mathrm{a}_{\mathrm{m}}\right)^{\prime}$ and $\omega=\left(\omega_{1}, \cdots, \omega_{\mathrm{m}}\right)^{\prime}$ be the vectors of machine ages and preventive maintenance rates respectively. The process $\zeta(\mathrm{t})$ is modelled by a continuous time Markov chain defined by machine ages and control dependent transition rates matrix $\mathrm{Q}(\cdot)$ defined as follows:

$$
\mathrm{Q}(\mathrm{a}, \omega)=\left\lfloor\lambda_{\alpha \beta}(\mathrm{a}, \omega)\right\rfloor
$$

with

$$
\sum_{\beta=1}^{\mathrm{M}} \lambda_{\alpha \beta}(\mathrm{a}, \omega)=0, \quad \alpha=1, \cdots, \mathrm{M}, \quad \text { with } \mathrm{M}=\operatorname{card}(\mathrm{B})
$$

The transition rates $\lambda_{\alpha \beta}(\cdot)$ are derived from the combination of those of the dependent processes $\zeta_{\mathrm{i}}(\mathrm{t}), \mathrm{i}=1, \cdots, \mathrm{m}$.

Our approach is used when the rate of change in the machine states is much higher than the rate at which the cost is discounted. In this paper, we assume a constant demand rate without any loss of generality. Two time scales are then considered: the discounting cost event and the machine state process time scales. When the difference between the two time scales is very large, the time can be split. Thus, the transition rates for the system $\lambda_{\alpha \beta}(\cdot)$ can be expressed as $\varepsilon^{-1} \mathrm{q}_{\alpha \beta}(\cdot)$, where $\mathrm{q}_{\alpha \beta}($.$) and the discount rates are of the same magnitude. The singular perturbation$ parameter $\varepsilon$ is used here to express the hierarchical structure of the proposed approach. With $\varepsilon<<1$, an equivalent deterministic problem can be derived from the formulation of the initial stochastic problem. Both control problems (stochastic and deterministic) are described by dynamic programming equations (DPE) presented later in this section. In order to increase the system capacity or the availability of the machines, we assume that the transition rate from the operational mode to the preventive maintenance mode for each machine is a control variable called $\omega_{i}(t), i=1, \cdots, m$.

The system behaviour is described by a hybrid state comprising both a discrete and a continuous component. The discrete component consists of the discrete event stochastic process 
$\zeta^{\varepsilon}(\mathrm{t})$, while the continuous component consists of continuous variables $\mathrm{x}=\left(\mathrm{x}_{1}, \cdots, \mathrm{x}_{\mathrm{n}}\right)^{\prime}$ and $\mathrm{a}=\left(\mathrm{a}_{1}, \cdots, \mathrm{a}_{\mathrm{m}}\right)^{\prime}$ corresponding to the inventory/backlog of products and the cumulative ages of machines. These state variables are described by the following differential equations:

$$
\begin{array}{rl}
\dot{\mathrm{x}}(\mathrm{t})=\mathrm{u}(\mathrm{t})-\mathrm{d} & \mathrm{x}(0)=\mathrm{x} \\
\dot{\mathrm{a}}(\mathrm{t})=\mathrm{f}(\mathrm{u}(\mathrm{t})) & \mathrm{a}(0)=\mathrm{a}
\end{array}
$$

where $x, a$ and $d$ are given initial surplus or backlog, initial machine ages and demand rates vectors respectively. Let $\widetilde{\mathrm{x}}=(\mathrm{x}, \mathrm{a})$ and $\widetilde{\mathrm{u}}=(\mathrm{u}, \omega)$. Let $\mathrm{u}=\left(\mathrm{u}_{1}, \cdots, \mathrm{u}_{\mathrm{n}}\right)^{\prime}$ denote the vector of production rates. The set of feasible control policies $\kappa(\alpha)$, is given by:

$$
\mathrm{K}(\alpha)=\left\{\begin{array}{l}
(\mathrm{u}(\mathrm{t}), \omega(\mathrm{t})) \in \mathfrak{R}^{\mathrm{n}+\mathrm{m}}, \mathrm{u}_{\mathrm{i}}(\mathrm{t}) \geq 0, \sum_{\rho=1}^{\mathrm{n}} \mathrm{u}_{\mathrm{p}}(\mathrm{t}) \gamma_{\mathrm{p}} \leq \mathrm{z}, \quad \mathrm{u}_{\mathrm{p}}=\sum_{\mathrm{i}=1}^{\mathrm{m}} \mathrm{u}_{\mathrm{ip}} \\
\text { and } 0 \leq \omega_{\mathrm{i}}(\mathrm{t}) \leq \omega_{\max }^{\mathrm{i}}
\end{array}\right\} \quad 0 \leq \mathrm{i} \leq \mathrm{m}
$$

where $\gamma_{\mathrm{p}}$ is the processing time of the part type $\mathrm{p}$, and $\omega_{\max }$ is the maximum preventive maintenance rate of each machine.

Let $\mathrm{G}(\alpha, \widetilde{\mathrm{x}}, \widetilde{\mathrm{u}})$ be the instantaneous cost defined as follows:

$$
\mathrm{G}(\alpha, \tilde{\mathrm{x}}, \tilde{\mathrm{u}})=\mathrm{c}^{+} \mathrm{x}^{+}+\mathrm{c}^{-} \mathrm{x}^{-}+\mathrm{c}^{\alpha}, \quad \forall \alpha \in \mathrm{B}
$$

where $\mathrm{c}^{+}$and $\mathrm{c}^{-}$are costs incurred per unit produced parts for positive inventory and backlog respectively, $\mathrm{x}^{+}=\left(\max \left(0, \mathrm{x}_{1}\right), \ldots, \max \left(0, \mathrm{x}_{\mathrm{n}}\right)\right)^{\prime}, \mathrm{x}^{-}=\left(\max \left(-\mathrm{x}_{1}, 0\right), \ldots, \max \left(-\mathrm{x}_{\mathrm{n}}, 0\right)\right)^{\prime}$ and $\mathrm{c}^{\alpha}$ are given constants used here for preventive maintenance and repair activity costs.

Our objective is to control the production rate $\mathrm{u}(\cdot)$ and the preventive maintenance rate $\omega(\cdot)$ (or the control policy $\widetilde{\mathrm{u}}(\cdot)$ ) so as to minimize the expected discounted cost given by:

$$
\mathrm{J}^{\varepsilon}(\alpha, \widetilde{\mathrm{x}}, \mathrm{u})=\mathrm{E}\left\{\int_{0}^{\infty} \mathrm{e}^{-\mathrm{pt}} \mathrm{G}(\alpha, \widetilde{\mathrm{x}}, \mathrm{u}) \mathrm{dt} \mid \mathrm{x}(0)=\mathrm{x}, \mathrm{a}(0)=\mathrm{a}, \varsigma^{\varepsilon}(0)=\alpha\right\}
$$

subject to constraints given by equations (1) to (4). The value function of such a problem is:

$$
v^{\in}(\alpha, \widetilde{\mathrm{x}})=\inf _{\widetilde{\mathrm{u}} \in \mathrm{K}(\alpha)} \mathrm{J}^{\in}(\alpha, \widetilde{\mathrm{x}}, \widetilde{\mathrm{u}}) \quad \forall \alpha \in \mathrm{B}
$$

The value function given by equation (6) is locally Lipschitz, convex and is the unique viscosity solution of the following HJB equations (see Kenne (1997) and Sethi and Zhang (1994))

$$
\rho v^{\epsilon}(\alpha, \tilde{x})=\min _{\tilde{u} \in \mathrm{K}(\alpha)}\left\{f(.) v_{x}^{\epsilon}(\alpha, \tilde{x})+G(\alpha, \tilde{x}, \tilde{u})+\frac{1}{\in} \sum_{\beta=1}^{m} q_{\alpha \beta} v^{\epsilon}(\beta, \tilde{x})\right\}
$$


where $f(\cdot)=(u-d, f(u))^{\prime}$ and $\mathrm{v}_{\mathrm{x}}^{\varepsilon}(\alpha, \widetilde{\mathrm{x}})$ is the gradient of $\mathrm{v}^{\varepsilon}($.$) in \widetilde{\mathrm{x}}$.

$$
\operatorname{dim}=p \prod_{i=1}^{n+m}\left(\frac{T_{u p}^{i}-T_{l o}^{i}}{h_{i}}+1\right) \times 3^{m+n} \times 2^{m}
$$

where $\mathrm{T}_{\text {up }}^{\mathrm{i}}, \mathrm{T}_{\text {lo }}^{\mathrm{i}}$ and $\mathrm{h}_{\mathrm{i}}$ are the upper value, the lower value and the discrete step of the state variable $T^{i}$. For each product $j, j=1, \ldots, n$, the production rate $u_{i j}, i=1, \ldots, m$, has three possible values $\left(0, \widetilde{d}_{i}^{j}, U_{i j}^{m}\right)$; this corresponds to $3^{m+n}$ points for the set of $u_{i j}$. For each machine, the preventive maintenance rate is chosen between two values, 0 and $\omega_{\mathrm{j}}^{\max }$; this gives $2^{\mathrm{m}}$ points for the $\mathrm{m}$ machines. The dimension $\operatorname{dim}$, as in equation (8), is very large for a multiple-part, multiplemachine manufacturing system. Given that there is no way to solve HJB equations (7) analytically, numerical methods based on dim are usually used to characterise the optimal control policy. A singular perturbation approach is used to define a hierarchical control scheme based on limiting probabilities and deterministic optimality conditions, as presented here in the case of identical machines. Such an approach is unusable in the case of non-identical machine manufacturing systems.

For identical machines, let $\left\{\mathrm{Z}^{\varepsilon}(\mathrm{t}), \mathrm{t} \geq 0\right\}$ be a finite-state stochastic process corresponding to the number of operational machines at time $t$ with values in $\varepsilon=\{0,1, \cdots, \mathrm{m}\}$. Our objective is to control the production rate $\mathrm{u}(\mathrm{t})$ and the preventive maintenance rate $\omega(\cdot)$ (or the control policy $\widetilde{\mathrm{u}}(\cdot)=(\mathrm{u}(\cdot)))$ so as to minimize the expected discounted cost given by:

$$
\mathrm{J}^{\varepsilon}(\alpha, \widetilde{\mathrm{x}}, \mathrm{u})=\mathrm{E}\left\{\int_{0}^{\infty} \mathrm{e}^{-\mathrm{pt}} \mathrm{G}(\alpha, \widetilde{\mathrm{x}}, \mathrm{u}) \mathrm{dt} \mid \mathrm{x}(0)=\mathrm{x}, \mathrm{a}(0)=\mathrm{a}, \mathrm{Z}^{\varepsilon}(0)=\alpha\right\}
$$

The value function of the control problem considered is given by:

$$
\mathrm{v}^{\in}(\alpha, \widetilde{\mathrm{x}})=\inf _{\widetilde{\mathrm{u}} \in \mathrm{K}(\alpha)} \mathrm{J}^{\in}(\alpha, \widetilde{\mathrm{x}}, \widetilde{\mathrm{u}}) \quad \forall \alpha \in \varepsilon
$$

Such a function is also locally Lipschitz, convex and is the unique viscosity solution of the following HJB equations:

$$
\rho v^{\epsilon}(\alpha, \widetilde{x})=\min _{\widetilde{u} \in K(\alpha)}\left\{(\mathrm{u}-\mathrm{d}) v_{\mathrm{x}}^{\in}(\alpha, \widetilde{\mathrm{x}})+\mathrm{f}(\mathrm{u}) \mathrm{v}_{\mathrm{a}}^{\in}(\alpha, \widetilde{\mathrm{x}})+\mathrm{G}(\alpha, \widetilde{\mathrm{x}}, \widetilde{\mathrm{u}})+\frac{1}{\in} \sum_{\beta=0}^{\mathrm{m}} \mathrm{q}_{\alpha \beta} v^{\in}(\beta, \widetilde{\mathrm{x}})\right\} \quad \forall \alpha \in \varepsilon
$$

The optimal control policy $\left(\mathrm{u}^{*}(\cdot), \omega^{*}(\cdot)\right)$ is the solution of HJB equations described by (11). Given the large size of (11) for multiple-machine, multiple-part type manufacturing systems, it is necessary here to proceed to the reduction of the system size through the limiting control problem. Such a problem is based on the stationary distribution of the stochastic process, which is computed here in terms of the mean values of the machine ages and preventive maintenance rates denoted by $a_{M}$ and $\bar{\omega}$ respectively. If $a_{i}$ and $\omega_{i}, i=1, \ldots, m$ are replaced in the model by $a_{M}$ and $\bar{\omega}$, a constant transition rates matrix $\mathrm{Q}\left(\mathrm{a}_{\mathrm{M}}, \bar{\omega}\right)$ is obtained. The finite state Markov chain associated 
to such a matrix is then homogeneous, with stationary or limiting probabilities $v=\left(v_{1}, v_{2}, \cdots, v_{m}\right)^{\prime}$ given by:

$$
v Q(.)=0 \text { and } \sum_{i=1}^{m} v_{i}=1
$$

With these probabilities, the convergence property of the initial stochastic control problem to the limiting problem when $\varepsilon \rightarrow 0$ is established in Soner (1993).

Based on the hierarchical model presented in Kenne (1997) and Kenne and Boukas (2003) stating that limiting probabilities are obtained for given $\bar{\omega}$, the overall optimization problem can be described by the following deterministic HJB equations:

$$
\rho v(\widetilde{x})=\min _{0 \leq \bar{\omega} \leq \bar{k} \omega_{\max }}\left\{\min _{\bar{u} \in K(\bar{k}(.))}\left[(\bar{u}-d) v_{x}(\widetilde{x})\right]+\frac{1}{m}\left(\sum_{j=1}^{n} k_{j} d_{j}\right) v_{a_{M}}(\widetilde{x})+\bar{G}(\widetilde{x}, \bar{\omega})\right\}
$$

The structure of the stochastic control presented in Boukas and Haurie (1990) and Kenne (1997) provide a machine age-dependent control policy through the solution of (13). The construction of the corresponding stochastic control policy structure provides the following production and preventive maintenance policies:

- Production Control Policy: The structure of the optimal production policy could be given by:

$$
u_{j}(\widetilde{x})=\left\{\begin{array}{ccc}
u_{\max }^{j} & \text { si } & x_{j}(t)<Z_{j} \\
d_{j} & \text { si } & x_{j}(t)=Z_{j} \\
0 & \text { si } & x_{j}(t)>Z_{j}
\end{array}\right.
$$

where $Z_{j}$ is the threshold value of product $j$ and $u_{\max }^{\mathrm{j}}=\sum_{\mathrm{i}=1}^{\mathrm{m}} \mathrm{u}_{\max }^{\mathrm{ij}}$ with $\mathrm{u}_{\max }^{\mathrm{ij}}$ describing the maximum production rate of product $j$ on machine $i$ for a multiple-machine, multiple-product manufacturing system.

- Preventive Maintenance Policy: The structure of the optimal machine age-dependent preventive maintenance policy depends on the mean age value $a_{M i}$ of the machine $i$ and a parameter $\delta_{i}$, and is defined as follows:

$$
\omega_{i}(\widetilde{x})=\left\{\begin{array}{cc}
\omega_{\max }^{\mathrm{i}} \text { if } \mathrm{a}_{\mathrm{i}}(\mathrm{t})>\mathrm{a}_{\mathrm{Mi}}-\delta_{\mathrm{i}} \\
0 & \text { otherwise }
\end{array}\right.
$$

Note that if $\delta_{i}>0$, preventive maintenance actions are performed before the machine age reaches $\mathrm{a}_{\mathrm{Mi}}$; otherwise they are performed after $\mathrm{a}_{\mathrm{Mi}}$. The values and sign of $\delta_{\mathrm{i}}$ depend mainly on the instantaneous cost parameters defined in equation (4) for a given manufacturing system. For such a system, a machine mean age value $\mathrm{a}_{\mathrm{Mi}}$ is determined using simulation through off-line experiments. The following machine age-dependent breakdown probability distribution is used to describe the dynamics of any machine $i$. 


$$
\mathrm{P}\left(a_{i}\right)=1-\exp \left(-k_{i} a_{i}(t)\right)
$$

where $k_{i}, i=1, \cdots, m$, are given constants and the machine age $a_{i}(t)$ is defined as the number of parts produced since the last intervention on the machine (repair or preventive maintenance). A machine age increasing failure rate (IFR) is given by (16), with $\mathrm{k}_{\mathrm{i}}<<1$. A well-known IFR, such as Weibull, can be recovered by choosing suitable values for $\mathrm{k}_{\mathrm{i}}$. Note that the optimal control policy is well defined by parameters $Z_{j}$ and $\delta_{i}$, which we call here input factors, for a given $a_{M i}$. In the next section, we present a heuristical control approach used here to estimate the optimal values of such factors. The proposed approach is based on a combination of analytical and simulation models, experimental design and response surface methodology.

\section{Control Approach}

Results obtained from traditional methods of production and preventive maintenance scheduling of multiple-machine manufacturing systems are not generally enough to provide a comfortable level of desired performance. To improve these methods, the descriptive capacities of conventional simulation models are combined with analytical models, experimental design and response surface methodology. This approach has been successfully used in the cases of singlemachine and multiple-identical-machine manufacturing systems (see Kenne and Gharbi (1999) and Gharbi and Kenne (2000)). A block diagram of the resulting control approach is depicted in Fig.1.

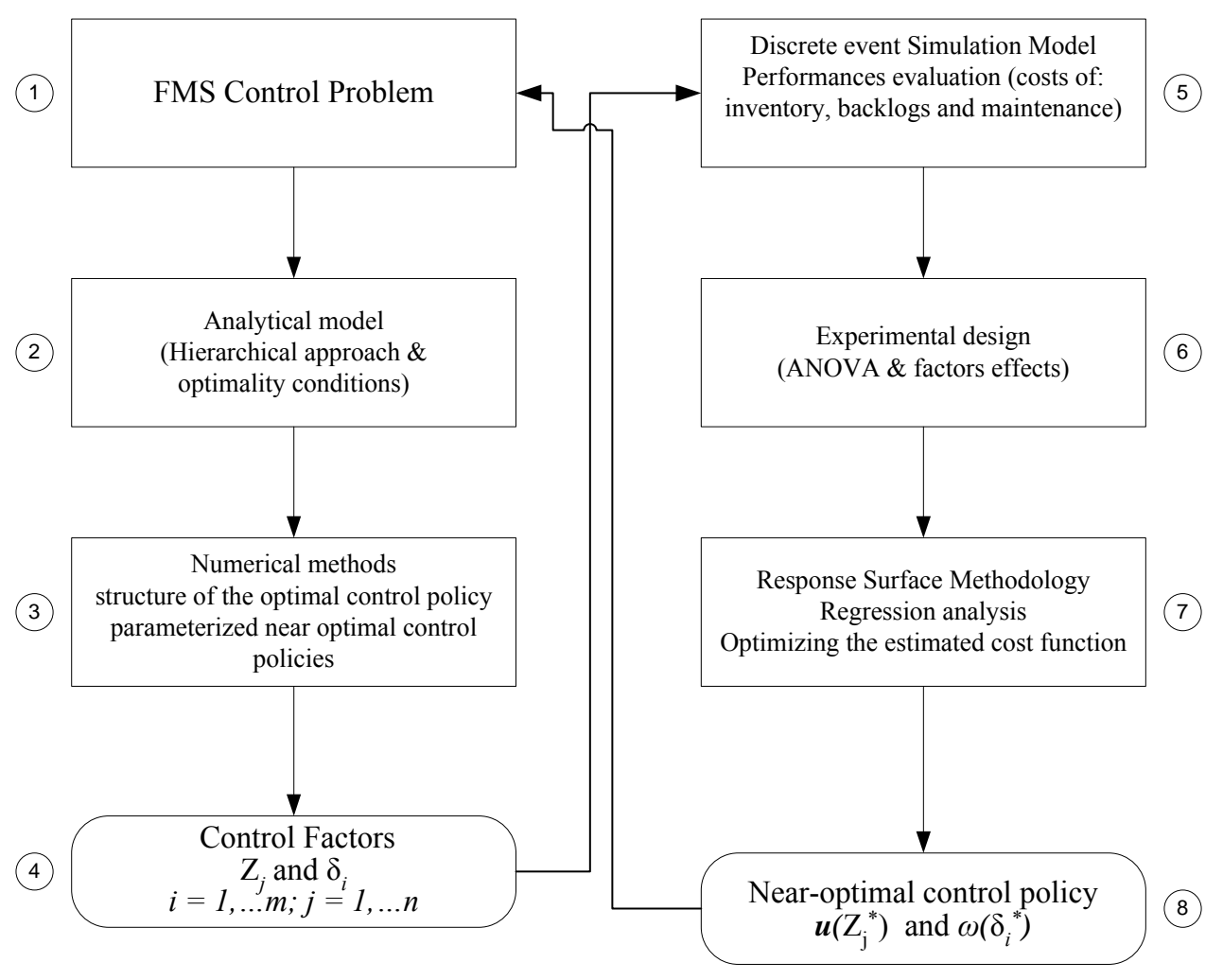

Fig.1: Proposed control approach 
The structure of the proposed control approach presented in Figure 1, consists of the following sequential steps:

1. The Control problem statement of the manufacturing system, as in section 2, consists of the representation of the production planning and maintenance scheduling problem through a stochastic optimal control model based on control theory. Hence, the problem of the optimal flow control for the manufacturing system considered is described in this first step, which contains a specification of the objective of the study. That objective is to find the control variables $(\boldsymbol{u}, \omega)$ called the production and preventive maintenance rates in order to improve the related output (i.e., the incurred cost).

2. The optimality conditions, described by the HJB equations, are obtained from the problem statement of the previous step. It is shown in this step that the value function, representing the incurred cost, is the solution of the HJB equations, and the corresponding control policy (production and preventive maintenance rates) is optimal. When the rate of change in the machine states is much higher than the rate at which the cost is discounted, the time can be split, and the singular perturbation approach used to develop optimality conditions for both stochastic and deterministic control problems. The control policy of the stochastic problem is constructed from that one of the corresponding deterministic problem.

3. The numerical methods are used in this step to solve the HJB equations of the limiting problem, given that there is no way of solving the equations analytically when it comes to real manufacturing systems (multiple-machine, multiple parts).

4. The control factors $\mathrm{Z}_{j}, j=1, \ldots n$ for production planning and $\delta_{i}, i=1, \ldots m$ for preventive maintenance scheduling, describe the numerical control policy obtained, extended to the stochastic problem.

5. The simulation model uses the near optimal control policy defined in the previous step as input for conducting experiments in order to evaluate the performances of the manufacturing system. Hence, for given values of the control factors, the cost incurred is obtained from the simulation model presented in section 4.

6. The experimental design approach defines how the control factors can be varied in order to determine the effects of the main factors and their interactions (i.e., analysis of variance or ANOVA) on the cost through a minimal set of simulation experiments.

7. The response surface methodology is then used to obtain the relationship between the incurred cost and significant main factors and interactions given in the previous step. The obtained regression model is then optimized in order to determine best values of factors called here $Z_{j}^{*}$ for production, and $\delta_{i}{ }^{*}$ for preventive maintenance scheduling.

8. The near-optimal control policy $\left(\boldsymbol{u}\left(\mathrm{Z}_{j}^{*}\right), \omega\left(\delta_{i}{ }^{*}\right)\right)$ is then an improved age-dependent hedging point policy to be applied to the manufacturing system. The application of the proposed control approach gives the production and preventive maintenance rates described by equations (14) and (15) respectively for best values of factors $Z_{j}{ }^{*}$ and $\delta_{i}{ }^{*}$.

\section{Simulation Model}

A discrete event simulation model that describes the dynamics of the system (1)-(2), is developed using the Visual SLAM language (Pritsker and O'Reilly, 1999). This model consists of several networks, each of which describes a specific task in the system (i.e., demand generation, control policy, states of the machines, inventory control..., etc.). The diagram of the proposed simulation model is shown in Fig. 2 with the following notation block descriptions: 


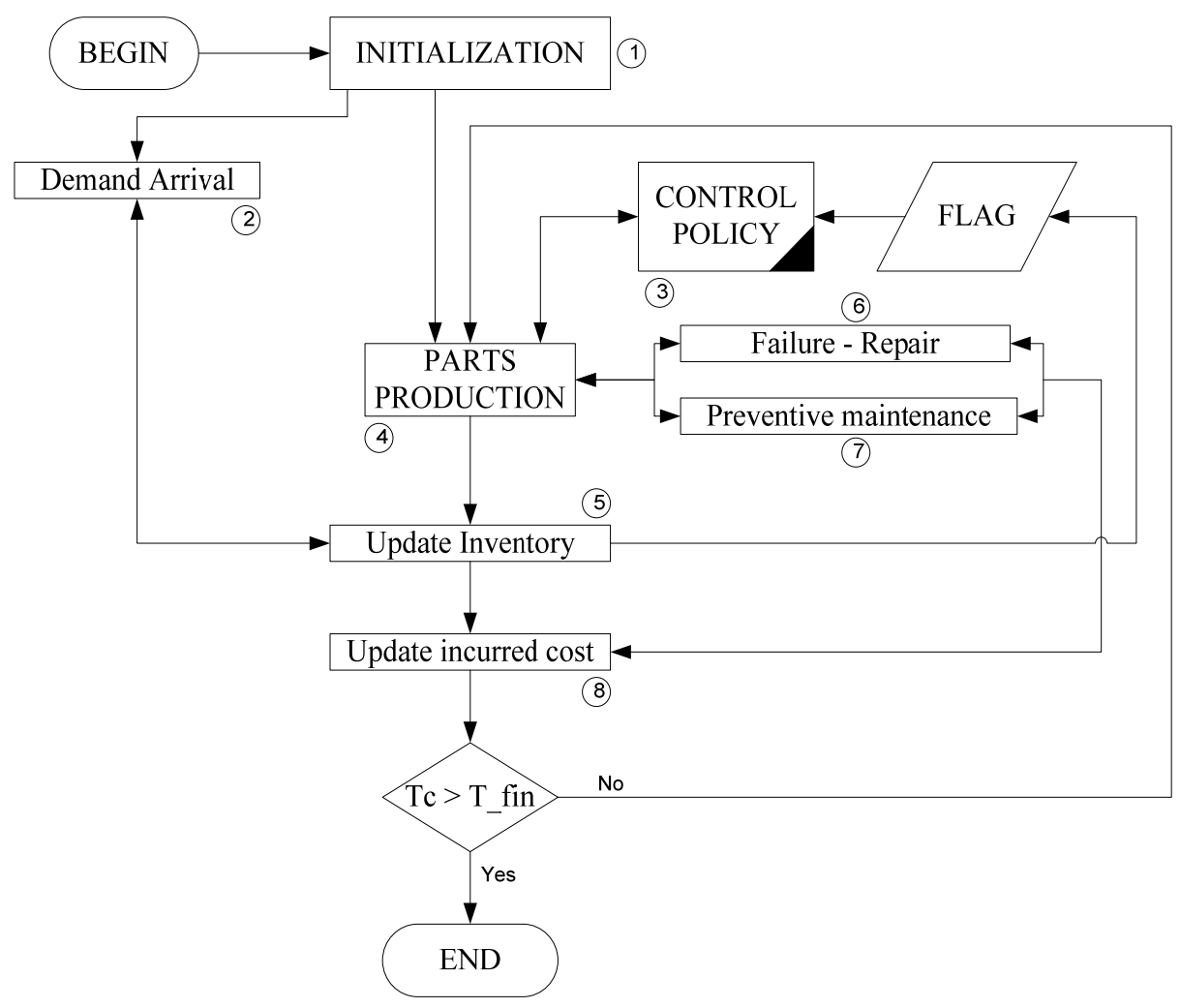

Fig. 2: Diagram of simulation model

1. The INITIALIZATION block initializes the variables (current surplus, production rates, incurred cost,...etc)

2. The Demand Arrival block performs the arrival of a demand for product $j$ at each $\mathrm{d}_{\mathrm{j}}^{-1}$ unit of time. A verification is then performed on the inventory value of product $j$, and the inventory or the backorder is updated.

3. The CONTROL POLICY segment block is defined in the previous section (see equation (14) for the machine production rates). The control policy is defined by the output of the FLAG block. This block is used to permanently verify the variation in the stock level $x_{j}(t)$. If $x_{j}(t)>Z_{j}$, then the production rate is set to a zero value; otherwise the production rate is set to the demand rate $\left(x_{j}(t)=Z_{j}\right)$ or to the maximum production rate $\left(x_{j}(t)<Z_{j}\right)$.

4. The PARTS PRODUCTION block performs the production of finished goods.

5. The update the inventory block performs the variation of the inventory level when a finished goods production or a demand arrival occurs (i.e. production of finished goods increases inventory if there is no backorder or it satisfies the cumulative demands, and hence decreases backorders). Off-line runs of the simulation model, for a two-different-machine, one-part type manufacturing system, using control policy described by (14) for $Z_{j}=20$ is illustrated in Fig. 3 for a product stock trajectory. 


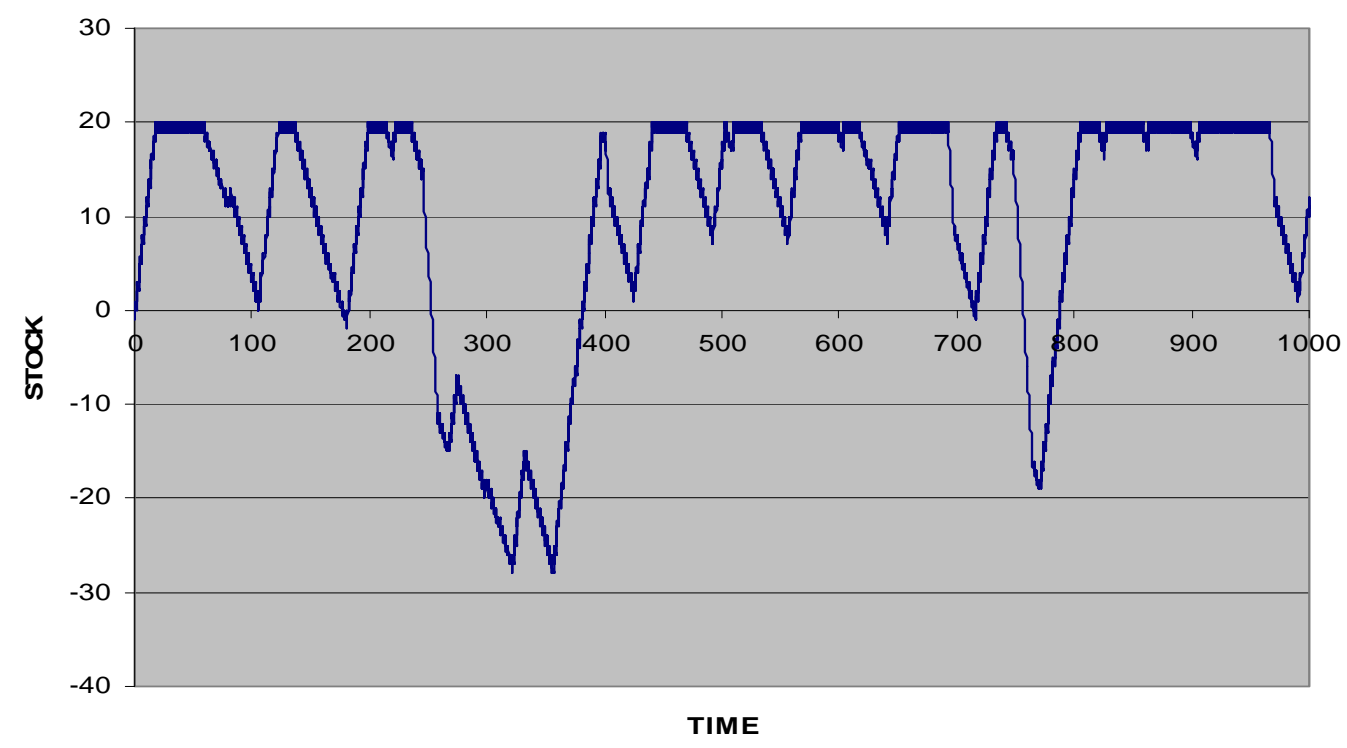

Fig. 3: A product stock trajectory $\left(\mathrm{Z}_{\mathrm{j}}=20\right)$.

It is interesting to note that: (i) the inventory level increases to $Z_{j}$ and remains at this value; (ii) the inventory level decreases during repair or preventive maintenance times; (iii) the decreasing level of the inventory depends on the repair and the preventive maintenance times, which are different for each machine.

6. The failure-repair block performs two functions: it defines the time-to-failure of each machine, and repairs broken ones. Using the breakdown probability distribution defined by (16), in Fig. 4, we present the machine age trajectories for two machines $\left(\mathrm{k}_{1}=10^{-4}, \mathrm{k}_{2}=2.10^{-4}\right)$, obtained from off-line runs of the simulation model. For example, machine 1, from initial time to the first jump time (where the breakdown occurs), the machine age increases from zero to 65 . The machine age is then set to zero during the repair time. When the machine is repaired, it produces parts and its age increases again.

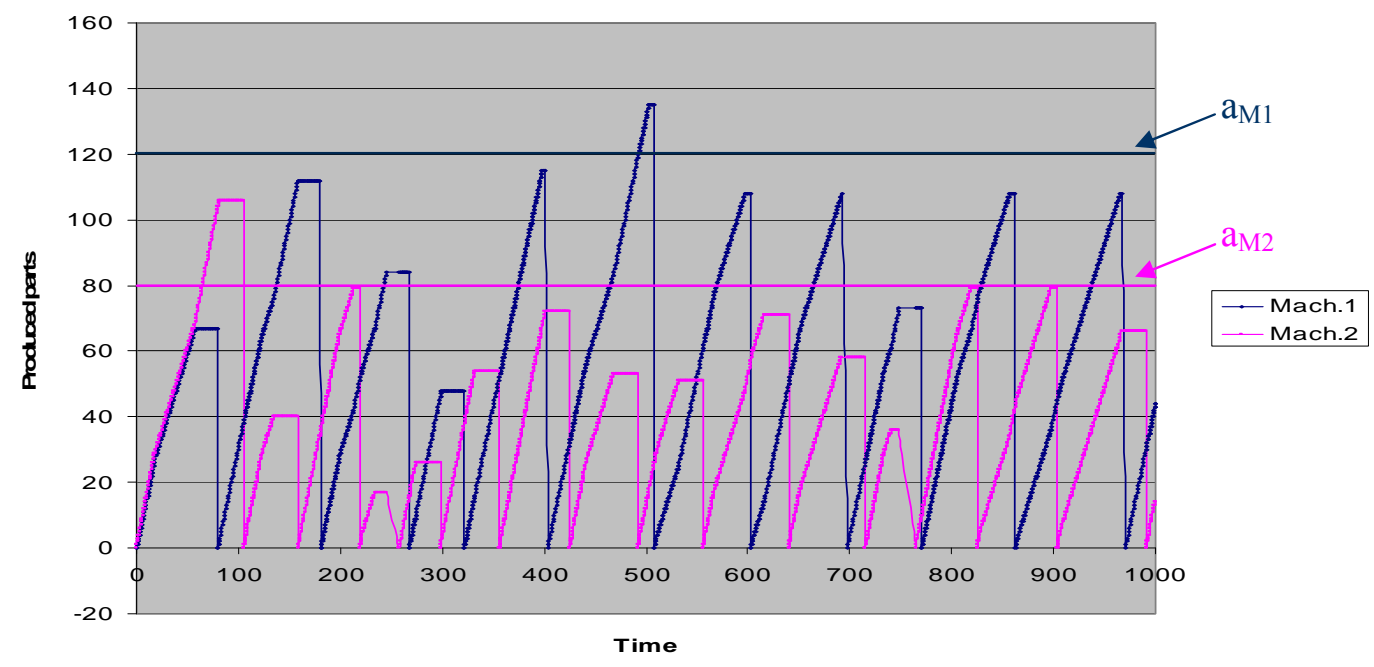

Fig. 4: Machine age trajectories 
7. The preventive maintenance block defines the time at which we should send each machine out for preventive maintenance. The mean value of the ages at which machine $i$ breakdowns occur is the mean age of machine $i$ denoted here as $\left(a_{M i}\right)$. The preventive maintenance on that machine should be done around that age (see equation (15) for the machine preventive maintenance rates). We determine $a_{M i}$ through off-line simulation runs. For the example illustrated in Fig. 4, these values are: $a_{M 1}=128$ and $a_{M 2}=89$. The failure-repair, the preventive maintenance and the update the inventory blocks update the incurred cost block.

8. The updates the incurred cost block calculates in a real time the cost of inventory, backlogs and corrective and preventive maintenance.

\section{Experimental Design and Response Surface Methodology}

To illustrate the approach presented in this paper, we consider a two-machine, one-product manufacturing system.

- The optimal flow control for the manufacturing system considered is formulated as in section 2, with optimality conditions given by HJB equations (11) and (13) for the two levels of the proposed hierarchical approach.

- The optimal control policy is approximated by a heuristic control policy defined in terms of design factors, as in equations (14)-(15) for the machine production and preventive maintenance rates. The objective of the proposed approach is to find the best parameters of the control variables $u($.$) and \omega($.$) , (i.e., Z_{1}, \delta_{1}$ and $\left.\delta_{2}\right)$, in order to improve the related output (i.e., the incurred cost).

- The simulation model describes the dynamics of the system using the control policy parameterized by the factors $Z_{1}, \delta_{1}$ and $\delta_{2}$. These factors are considered as the input of such a model, and the corresponding incurred cost is defined as its output.

- From the values of the input factors and the corresponding cost values, the experimental design approach determines input factors and/or their interactions that have significant effects on the output.

Significant factors or interactions are then considered as input of a response surface methodology, in order to fit the relationship between the cost and the input factors. The optimal values of the input factors, called $\mathrm{Z}_{1}{ }^{*}, \delta_{1}{ }^{*}$ and $\delta_{2}{ }^{*}$ are determined from this estimated relation. The related machine age-dependent modified hedging point policy is then an improved hedging point policy to be applied to the manufacturing system.

Due to the convexity of the value function (see section 2), we selected a $3^{3}$ response surface design. The experimental design is used to study and understand the effects that some parameters, namely $Z_{1}, \delta_{1}$ and $\delta_{2}$, for the manufacturing system, have on the performance measure (i.e., the cost).

\subsection{Numerical Example}

The following are the numerical values of the constants used previously: $\mathrm{d}=2 ; U_{\max }^{1}=1.5$; $U_{\max }^{2}=1.6 ; \mathrm{c}^{+}=1 ; \mathrm{c}^{-}=10 ; \mathrm{c}^{2}{ }_{1}=60 ; \mathrm{c}^{2}{ }_{2}=50 ; \mathrm{c}^{3}{ }_{1}=100 ; \mathrm{c}^{3}{ }_{2}=80 ; \mathrm{q}_{12}{ }^{1}=0.015 ; \mathrm{q}_{12}{ }^{2}=0.022 ; \mathrm{q}_{21}{ }^{1}=0.045$; $\mathrm{q}_{21}{ }^{2}=0.042 ; \mathrm{q}_{31}{ }^{1}=0.181 ; \mathrm{q}_{31}{ }^{2}=0.167 ; \mathrm{k}_{1}=10^{-4} ; \mathrm{k}_{2}=2.10^{-4}$. Note that, in the case of non-identical machines, $\mathrm{c}^{2}{ }_{\mathrm{i}}$, and $\mathrm{c}^{3}{ }_{\mathrm{i}}$ are used as repair and maintenance costs of machine $\mathrm{i}$, respectively. In 
addition, $\mathrm{q}_{12}{ }^{\mathrm{i}}$ is the breakdown rate of machine $\mathrm{i}$, and $\mathrm{q}_{21}{ }^{\mathrm{i}}$ and $\mathrm{q}_{31}{ }^{\mathrm{i}}$ are respectively the corrective and preventive maintenance rates for machine i. Based on off-line simulation runs, where the minimum and the maximum values of the factors were observed, the independent variable levels were chosen as in Table 1.

Table 1:

Level of independent variables

\begin{tabular}{cllll}
\hline Factor & Low Level & Center & High Level & Description \\
\hline $\mathrm{Z}_{1}$ & 10 & 30 & 50 & Stock level \\
$\delta_{1}$ & -80 & 0 & +80 & Maintenance for $\mathrm{M}_{1}$ \\
$\delta_{2}$ & -60 & 0 & +60 & Maintenance for $\mathrm{M}_{2}$ \\
\hline
\end{tabular}

Three replications were conducted for each combination of the factors, and therefore, $81\left(3^{3} \times 3\right)$ simulation runs were made. To reduce the number of replications, we used a variance reduction technique called common random numbers (Law and Kelton (2000)). We conducted some preliminary simulation experiments using 3 replications, and noticed that the variability allows the effects to be distinguished.

\subsection{Result Analysis}

The statistical analysis of the simulation data consists of the multifactor analysis of variance (ANOVA). This is done using a statistical software application, such as STATGRAPHICS, to provide the effects of the three independent variables $\left(Z_{1}, \delta_{1}\right.$ and $\left.\delta_{2}\right)$ on the dependant variable (Cost). The ANOVA table corresponding to the generated data is illustrated in Table 2. From Table 2, as all the p-values are less than $5 \%$, we conclude that the main factors $Z_{1}, \delta_{1}$ and $\delta_{2}$, their quadratic effects, as well as their interactions are significant at the 0.05 level. The R-squared value of 0.9414 from the ANOVA table, states that $94 \%$ of the total variability is explained by the model (Montgomery (2001)).

Table 2:

ANOVA Table

\begin{tabular}{llllll}
\hline Source & Sum of squares & Df & Mean Square & F-Ratio & P-Value \\
\hline A:Z1 & 2725.45 & 1 & 2725.45 & 458.53 & 0,0000 \\
B: d1 & 80.0371 & 1 & 80.0371 & 13.47 & 0.0005 \\
C:d2 & 503.709 & 1 & 503.709 & 84.74 & 0.0000 \\
AA & 1608.54 & 1 & 1608.54 & 270.62 & 0.0000 \\
AB & 583.469 & 1 & 583.469 & 98.16 & 0.0000 \\
BB & 72.2547 & 1 & 72.2547 & 12.16 & 0.0009 \\
BC & 97.0614 & 1 & 97.0614 & 16.33 & 0.0001 \\
CC & 219.716 & 1 & 219.716 & 36.97 & 0.0000 \\
Blocks & 38.8702 & 2 & 19.4351 & 3.27 & 0.0440 \\
Total error & 410.125 & 69 & 5.94384 & & \\
Total (corr.) & 6994.06 & 80 & & & \\
R-squared =94.1361 percent & & & & \\
\hline
\end{tabular}


The residual analysis was used to verify the adequacy of the model. A residual versus predicted value plot and normal probability plot were used to test the homogeneity of the variances and the residual normality, respectively. We conclude that the model is satisfactory, and there is no need for the transformation of response variables or for additional replications for the simulation model. The second order model is then given by:

$$
\begin{aligned}
\text { Cost }=\quad 63.928 & -1.063 Z_{1}-0.060 \delta_{1}-0.056 \delta_{2}+0.024 Z^{2}{ }_{1}+0.0025 Z_{1} * \delta_{1} \\
& +0.0036 Z_{1} * \delta_{2}+0.00031 \delta_{1}{ }^{2}+0.00034 \delta_{1} * \delta_{2}+0.00097 \delta_{2}{ }^{2}
\end{aligned}
$$
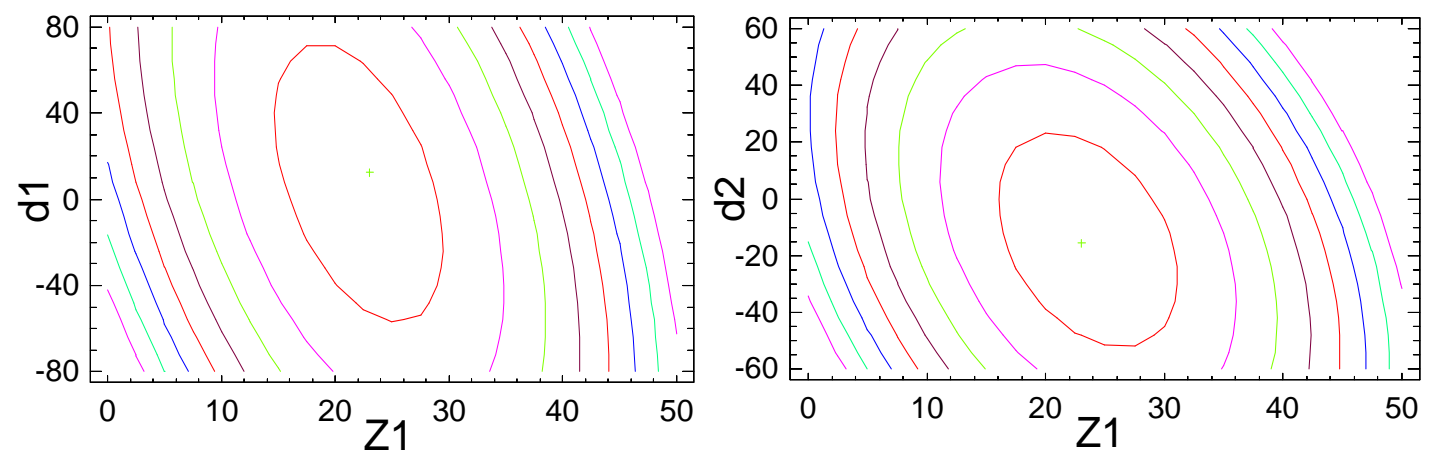

Fig. 5: Estimated response surface

The near-optimal control policy to be applied to the manufacturing system considered is defined by the minimum of the cost function (17) located at $Z_{1}{ }^{*}=22.99, \delta_{1}{ }^{*}=12.36$ and $\delta_{2}{ }^{*}=-$ 15.59 as shown in Fig. 5. A cost value of 51.76 is obtained with such a control policy.

To crosscheck the validity of the solution, $Z_{1}{ }^{*}=22.99, \delta_{1}{ }^{*}=12.36$ and $\delta_{2}{ }^{*}=-15.59$ were used as input to the simulation model. The cost value obtained was 51.16, which falls in the $95 \%$ confidence interval $\left(\bar{X}(n) \pm t_{n-1,1-\frac{\alpha}{2}} \sqrt{\frac{S^{2}(n)}{n}}=[50.39 ; 51.93]\right)$, obtained using $\mathrm{n}=10$ replications of

the simulation model. $Z_{1}{ }^{*}, \delta_{1}{ }^{*}$ and $\delta_{2}{ }^{*}$ define the best-modified hedging point policy to be applied to the manufacturing system considered. With the aforementioned optimal values of the independent factors or input parameters, the cost is minimized and the corresponding control policy is the best approximation of the optimal control one. The following control policy is to be applied to the manufacturing system presented in this example:

- If inventory level of product 1 is greater than 23 , then the production rate is set to zero value;

- If inventory level of product 1 is equal to 23 , then produce at the demand rate;

- If inventory level of product 1 is less than 23 , then produce at the maximum production rate.

- If the age of machine 1 is greater than $115.64\left(\mathrm{a}_{\mathrm{M} 1}-\delta_{1}{ }^{*}=128-12.36\right)$, then send machine 1 to preventive maintenance if the inventory level of product 1 is equal to 23 .

- If the age of machine 2 is greater than $104.59\left(\mathrm{a}_{\mathrm{M} 2}-\delta_{2}{ }^{*}=89-(-15.59)\right)$, then send machine 2 to preventive maintenance if the inventory level of product 1 is equal to 23 . 


\subsection{Sensitivity Analysis.}

A set of numerical examples are considered on the sensitivity of the obtained control policy with respect to inventory, backlog, corrective and preventive maintenance costs (i.e., $\mathrm{c}^{+}, \mathrm{c}^{-}, \mathrm{c}^{2}{ }_{\mathrm{i}}$, $\mathrm{c}^{3}{ }_{\mathrm{i}}$ ). The following variations, illustrated in Table 3, are explored and compared to a basic case.

- Decreasing $\mathrm{c}^{+}$: this must result in a tendency to increase the stock level in order to avoid further backlog costs.

- Increasing $\mathrm{c}^{+}$: this must result in a tendency to decrease the stock level in order to avoid further inventory costs.

- Decreasing $\mathrm{c}^{-}$: this must result in a tendency to decrease the stock level in order to avoid further inventory costs.

- Increasing $c^{-}$: this must result in a tendency to increase the stock level in order to avoid further backlog costs.

- Decreasing $\mathrm{c}^{2}{ }_{\mathrm{i}}$ (corrective maintenance cost): this must result in a tendency to delay the preventive maintenance period (fewer preventive maintenance activities).

- Increasing $\mathrm{c}^{2}{ }_{\mathrm{i}}$ (corrective maintenance cost): this must result in a tendency to advance the preventive maintenance period (more preventive maintenance activities).

- Decreasing $\mathrm{c}^{3}$ (preventive maintenance cost): this must result in a tendency to advance the preventive maintenance period (more preventive maintenance activities).

- Increasing $\mathrm{c}^{3}{ }_{\mathrm{i}}$ (preventive maintenance cost): this must result in a tendency to delay the preventive maintenance period (fewer preventive maintenance activities).

Table 3:

Sensitivity analysis table

\begin{tabular}{ccccccccccc}
\hline $\mathrm{c}^{+}$ & $\mathrm{c}^{-}$ & $\mathrm{c}^{2}{ }_{1}$ & $\mathrm{c}^{2}{ }_{2}$ & $\mathrm{c}^{3}{ }_{1}$ & $\mathrm{c}^{3}{ }_{2}$ & $Z_{1}{ }^{*}$ & $\delta_{1}{ }^{*}$ & $\delta_{2}{ }^{*}$ & Cost $^{*}$ & Remark \\
\hline 1 & 10 & 60 & 50 & 140 & 120 & 22.99 & 12.36 & -15.58 & 51.76 & Basic case \\
$\mathbf{0 . 5}$ & 10 & 60 & 50 & 140 & 120 & $\mathbf{3 5 . 9 7}$ & -23.81 & -28.76 & 40.22 & $\mathrm{Z}_{1}{ }^{*}$ increases \\
$\mathbf{1 . 5}$ & 10 & 60 & 50 & 140 & 120 & $\mathbf{1 0 . 9 6}$ & 46.20 & -1.93 & 57.88 & $\mathrm{Z}_{1}{ }^{*}$ decreases \\
1 & $\mathbf{8}$ & 60 & 50 & 140 & 120 & $\mathbf{1 8 . 4 4}$ & 8.28 & -14.57 & 49.26 & $\mathrm{Z}_{1}{ }^{*}$ decreases \\
1 & $\mathbf{1 2}$ & 60 & 50 & 140 & 120 & $\mathbf{2 6 . 0 4}$ & 18.26 & -17.00 & 53.53 & $\mathrm{Z}_{1}{ }^{*}$ increases \\
1 & 10 & $\mathbf{5 5}$ & 50 & 140 & 120 & 23.32 & $\mathbf{5 . 6 2}$ & -15.17 & 51.12 & $\delta_{1}{ }^{*}$ decreases \\
1 & 10 & $\mathbf{6 5}$ & 50 & 140 & 120 & 22.63 & $\mathbf{1 9 . 8 1}$ & -16.04 & 52.38 & $\delta_{1}{ }^{*}$ increases \\
1 & 10 & 60 & $\mathbf{4 5}$ & 140 & 120 & 23.20 & 12.89 & $\mathbf{- 1 8 . 7 2}$ & 50.71 & $\delta_{2}{ }^{*}$ decreases \\
1 & 10 & 60 & $\mathbf{5 5}$ & 140 & 120 & 22.77 & 11.75 & $\mathbf{- 1 2 . 2 0}$ & 52.80 & $\delta_{2}{ }^{*}$ increases \\
1 & 10 & 60 & 50 & $\mathbf{1 3 0}$ & 120 & 22.25 & $\mathbf{2 7 . 8 1}$ & -16.65 & 51.44 & $\delta_{1}{ }^{*}$ increases \\
1 & 10 & 60 & 50 & $\mathbf{1 5 0}$ & 120 & 23.53 & $\mathbf{1 . 2 2}$ & -14.80 & 52.01 & $\delta_{1}{ }^{*}$ decreases \\
1 & 10 & 60 & 50 & 140 & $\mathbf{1 1 0}$ & 22.59 & 11.55 & $\mathbf{- 9 . 5 8}$ & 51.57 & $\delta_{2}{ }^{*}$ increases \\
1 & 10 & 60 & 50 & 140 & $\mathbf{1 3 0}$ & 23.3 & 12.90 & $\mathbf{- 1 9 . 9 9}$ & 51.92 & $\delta_{2}{ }^{*}$ decreases \\
\hline
\end{tabular}

Through the above analysis, it clearly appears that the results obtained make sense, and that the proposed approach is robust.

In the second part of this section, we will discuss how to control more complex manufacturing systems. For an $m$ machine, $n$ products manufacturing system as in this paper, we obtain an $m+n$ factors experimental design (i.e., one factor for each machine and product). For large values of $m$ and $n$, a more appropriate experimental design must be explored, since the complete $3^{m+n}$ is very difficult to implement. In such a situation, a two-step design approach is recommended: 
1. Use of two-level fractional factorial designs (i.e. $2^{\mathrm{f}-\mathrm{p}}$ ) as filter in order to eliminate nonsignificant factors and/or interactions (Montgomery, 2001).

2. Use of experimental design related to significant factors or interactions. The Box-Benhken or Box-Wilson central composite designs are commonly used at this level (Montgomery, 2001). However, the Box-Wilson design is preferred because we reuse all the results of the experiments performed during the screening step.

The proposed approach significantly reduces the number of simulation runs, and should gives rise to near-optimal control policies for more complex manufacturing systems.

\section{Conclusion}

In this paper, we have extended the concept of hedging point policy to the production and preventive maintenance control problem of a multiple, non-identical machine manufacturing system. The proposed approach was based on the combination of the hierarchical control model, simulation experiments, experimental design and RSM. First, we investigated a near-optimal control policy of a machine age dependent Markov process through the construction of the stochastic control policy from one of the dependant deterministic models. We then associated to such a policy parameters called independent variables. A simulation model was developed to describe the dynamic of the production system under the proposed modified hedging point policy. An experimental design approach was then used to investigate the effects of specific factors on the cost incurred during the production horizon. The proposed approach combines the simulation method with the statistical method to provide the estimation of the cost function related to the control problem considered. A response surface methodology was used to perform this function in terms of significant main factors and interactions given by the experimental design approach. From the estimation of the cost function, the best values of control parameters were easily computed.

\section{7. $\quad$ References}

Akella R. and Kumar P. R. (1986), Optimal Control of Production Rate in a Failure Prone Manufacturing System. IEEE Trans. On Automatic Control, Vol. AC-31, No. 2, pp. 116-126.

Boukas, E. K. and Haurie A. (1990) Manufacturing Flow Control and Preventive Maintenance: A Stochastic Control Approach. IEEE Transactions on Automatic Control, Vol 33 No 9, 1024-1031.

Boukas E.K., Kenne J.P. and Zhu Q. (1995) Age-dependent Hedging Point Policies in Manufacturing Systems. American Automatic Control Council, Seattle, Washington, June 21-23.

Gharbi A. and Kenne J. P. (2000) Production and Preventive Maintenance Rates Control for a Manufacturing System: An Experimental Design Approach. International Journal of Production Economics, Vol 65 No 3, pp 275-287.

Kenne J.P. (1997), Planification de la Production et de la Maintenance des Systèmes de Production: Approche Hiérarchisée. Ph.D thesis, Ecole Polytechnique de Montréal, Université de Montréal.

Kenne, J. and P. Boukas, E. K., (2003) Hierarchical Control of Production and Maintenance Rates in Manufacturing Systems. Journal of Quality in Maintenance Engineering, Vol. 9, No. 1. 
Kenne J.P. and Gharbi A. (1999) Experimental Design in Production and Maintenance Control of a Single Machine, Single Product Manufacturing System. International Journal of Production Research, 37(3), 621-637.

Kokotovic, P., Khalil, H. P. and O'Relly J. (1986) Singular perturbation methods in control: analysis and design, Academic Press.

Lehoczky J., Sethi S., Soner H.M. and Taksar M. (1991) An asymptotic Analysis of Hierarchical Control of Manufacturing Systems Under Uncertainty. Mathematics of operations research, 16(3), 596-608.

LAW, A.M. and W.D. Kelton (2000) Simulation Modeling and Analysis. $3^{\text {ed }}$ edition, Mc GrawHill.

Montgomery, D. C. (2001) Design and analysis of experiments. $5^{\text {th }}$ edition, John Wiley \& Sons.

Older G.J. and Suri R. (1980) Time Optimal Part-Routing in a Manufacturing System with Failure Prone Machines. Proc. $19^{\text {th }}$ Conf. Decis. Contr. Albuquerque, NM.

Pritsker A. A. B and O'Reilly J. J. and LaVal D. K., Simulation with Visual SLAM and AweSim. John Wiley \& Sons, 1999.

Rishel Dynamic Programming and Minimum Principles for Systems with Jump Markov Disturbances. SIAM journal on Control, 13, 338-371.

Sethi S.P. and Zhang Q. (1994), Hierarchical Control Decision Making in Stochastic Manufacturing Systems. Birkhauser, Boston.

Soner H.M. (1993) Singular Perturbation in Manufacturing. SIAM J. Control and Optimization, $31(1), 132-146$. 